\title{
EL IMPACTO DE LA ESCRITURA ACADÉMICA EN LA FORMACIÓN A NIVEL DE POSTGRADO: EL CASO DE LA UNIVERSIDAD PEDAGÓGICA NACIONAL FRANCISCO MORAZÁN (UPNFM) DE HONDURAS
}

\author{
THE IMPACT OF ACADEMIC WRITING ON POSTGRADUATE \\ TRAINING: THE CASE OF THE FRANCISCO MORAZÁN \\ NATIONAL PEDAGOGICAL UNIVERSITY (UPNFM) OF \\ HONDURAS.
}

\begin{abstract}
Sandra Cáceres Estrada*
RESUMEN: Este trabajo presenta el análisis de la escritura académica producida por estudiantes de postgrado, entendida, como una de las principales habilidades básicas para la producción de conocimiento. Específicamente, se analiza el nivel de escritura académica de estudiantes de programas de maestría de la Facultad de Humanidades (FAHU), de la Universidad Pedagógica Nacional Francisco Morazán (UPNFM), en Honduras. Uno de los objetivos fundamentales de la investigación es analizar los rasgos constitutivos de la escritura y la competencia que evidencian los maestrantes, en la escritura de artículos, en relación con la producción de conocimiento. La investigación se fundamenta en la perspectiva teórica del enfoque pragmático textual, el análisis del discurso y de la lingüística textual, de manera que para el análisis de los datos fueron considerados diversos aspectos textuales, importantes para la producción de un texto escrito. Además, se presentan, de forma resumida, los resultados más relevantes del análisis y la interpretación de los mismos, así como las conclusiones; los cuales merecen la atención, por parte de autoridades universitarias, para la toma de algunas decisiones.
\end{abstract}

Palabras claves: Escritura académica; Enfoque pragmático; Lingüística textual; Análisis del discurso; Postgrado.

\begin{abstract}
This work presents the analysis of academic writing produced by graduate students, known as one of the main basic skills to produce knowledge. Specifically, the level of academic writing of students from the Faculty of Humanities master's programs, of the Francisco Morazán National Pedagogical University (UPNFM), in Honduras is analyzed. One of the fundamental objectives of the research is to analyze the constitutive features of writing and the competence that graduate students demonstrate, in the writing of articles, concerning the production of knowledge. The research is based on the theoretical perspective of the textual pragmatic approach, discourse analysis, and textual
\end{abstract}

\footnotetext{
*Universidad Pedagogica Nacional Francisco Morazán (Honduras).
} 
linguistics, so that for the data analysis, various textual aspects were considered, which are very important to produce a written text. In addition, the most relevant results of the analysis and their interpretation, as well as the conclusions, are presented in a summarized way, which deserves the attention of the university authorities, for making some decisions.

Keywords: Academic writing; Pragmatic approach; Textual linguistics; Discourse analysis; Postgraduate.

\section{INTRODUCCIÓN}

La escritura académica es uno de los más importantes desafíos que enfrentan los estudiantes, especialmente, en el nivel de postgrado. En tal sentido, las habilidades básicas de comprensión lectora y de expresión escrita son competencias que se deben fortalecer, a lo largo de los diversos niveles educativos, puesto que van de la mano y, además, según Cassany, Luna, y Sanz, (1997, pp. 268-270), su dominio presupone el manejo de diversas destrezas y microhabilidades tales como reproducir, analizar, organizar ideas, formular, reformular, redactar, revisar, rehacer, explicar. En consecuencia, si el estudiante de postgrado demuestra dominio de estas microhabilidades, tendrá la competencia para la escritura académica y, por lo tanto, para la producción de conocimiento, lo que asegurará su éxito, respecto a la redacción de su trabajo de tesis.

El presente artículo describe los resultados de la investigación sobre el impacto de la escritura académica en la formación, a nivel de postgrado: el caso de la UPNFM. La investigación se dirigió por los siguientes objetivos: por un lado, analizar e identificar si las producciones escritas por los maestrantes de la UPNFM cumplen con las diversas propiedades o cualidades discursivo-textuales de intencionalidad, adecuación, coherencia, unidad temática, progresión temática, cohesión, intertextualidad y gramaticalidad; para que los textos sean valorados como tal. Por otro lado, se analiza el tipo de relación que los maestrantes establecen respecto a la citación de otros autores, en sus producciones escritas; 
con el fin de valorar si realizan una citación adecuada, o, por el contrario, si cometen plagio. Asimismo, describir las distintas operaciones discursivas desarrolladas por los maestrantes, en sus producciones escritas e identificar si los maestrantes realizan un uso adecuado de los procedimientos de cohesión textual.

Todo lo anterior, con el objetivo de diagnosticar las fortalezas y debilidades que evidencian los maestrantes, en cuanto a la habilidad de escritura académica y producción de conocimiento, lo que incide, directamente, en la redacción de su tesis de grado, y, a la vez, influye, de manera indirecta, en el caso de que el maestrante no logre escribir su tesis, en la eficiencia terminal de los programas de maestría.

\section{CONTEXTUALIZACIÓN}

Situación de la UPNFM respecto a la escritura académica en el postgrado

La Universidad Pedagógica Nacional Francisco Morazán (UPNFM), a través de la Dirección de Postgrado, ofrece diversos programas de maestrías tanto de la Facultad de Humanidades como de la Facultad de Ciencia y Tecnología, con el propósito de fortalecer el nivel de educación superior del sistema educativo nacional y brindar una serie de opciones de crecimiento académico-profesional y de proyección nacional e internacional. Sin embargo, uno de los problemas que enfrenta la UPNFM acerca de los avances, por parte de los maestrantes, en estos programas de postgrado, es el bajo nivel de eficiencia terminal de los mismos, puesto que, a lo largo del tiempo, se ha observado que el número de estudiantes que logra culminar su proyecto de conclusión de grado, es decir, la tesis, es mínimo.

Debido a la situación descrita anteriormente, la Dirección de Postgrado emprendió un Proyecto de Fortalecimiento a la Eficiencia Terminal, con el fin de apoyar a aquellos egresados de los programas de maestría que, por una u otra razón, no consiguen culminar su tesis y, por consiguiente, no logran graduarse, tal como lo afirma la Dirección de Postgrado (2020, p. 5): 
Desde la Dirección de Postgrado y en conjunto con la Vicerrectoría de Investigación y Postgrado se analizó la situación de los egresados, observando que era mínimo el número de estudiantes que habían defendido sus trabajos de tesis, situación que despertó el interés para que el año 2015, previo a un diagnóstico de necesidades, se lanzara el proyecto de Fortalecimiento a la Eficiencia Terminal de los Programas de Postgrado de la UPNFM, el mismo se implementó con el propósito de que los egresados de los diferentes programas de maestría, finalizasen su programa de estudios, presentando y defendiendo su proyecto de tesis, y cumplir con el requisito para obtener el título y graduarse dentro de los plazos establecidos por la Institución.

El escenario detallado anteriormente, respecto a la problemática en mención, obedece a diversos factores que, de alguna manera, condicionan el óptimo desempeño de los maestrantes. Según el estudio acerca de la eficiencia terminal, realizado por la Dirección de Postgrado, esto se debe a aspectos personales, carga laboral, también, a aspectos académicos, tales como la deficiencia, en algunos casos, en cuanto al dominio metodológico en investigación. De igual manera, Medina Otero (2008), identificó en su trabajo que "el principal obstáculo que tienen los estudiantes para obtener el grado son las dificultades en la escritura, lo cual se refleja en la elaboración de su trabajo de tesis" (Dirección De Postgrado, 2020, p.8), este factor se suma a los mencionados anteriormente. Además, otro elemento importante, que incide, en ciertos casos de rezago, es la carencia de docentes asesores especialistas en algunas áreas disciplinares.

Al respecto, un claro ejemplo de rezago se evidencia en la evolución del proceso de avance de la primera promoción de la Maestría en Enseñanza de Lenguas, la cual inició en el tercer período académico del año 2004, en la misma ingresaron 26 estudiantes, de los cuales dos abandonaron el programa y 24 maestrantes culminaron el pénsum referente a los módulos que contiene el plan de estudios de la maestría en mención. Sin embargo, de esta promoción, solamente 12 maestrantes lograron graduarse, en cambio, 12 de ellos, a la fecha, no se graduaron, y el principal motivo es el no haber culminado su tesis de grado, lo que representa un porcentaje de rezago del 50\%. (Secretaría General, UPNFM, 2021). Esto es una evidencia muy explícita de la dificultad, por parte de los maestrantes, en la expresión escrita. En tal sentido, la UPNFM debe tomar 
medidas que contribuyan al fortalecimiento de la escritura académica, tal como sucede en otros países de Latinoamérica. Al respecto, en la Universidad Pedagógica Nacional (UPN) de México se realizó un estudio sobre "La atención de la escritura académica en el posgrado: una experiencia en la UPN", en el mismo se comenta que:

Para el caso de América Latina, en países como Argentina, Venezuela, Chile y Colombia, la composición escrita y la redacción académica, son tareas que se encuentran incorporadas curricularmente a los diversos programas educativos. La composición escrita en la universidad es una responsabilidad de todos los docentes. En México, sigue siendo una tarea pendiente. (Cruz Martínez, 2014-2015, p. 52)

Es meritorio resaltar, que, además de los países mencionados anteriormente, Brasil realiza grandes esfuerzos sobre el tema en cuestión, a través de sus universidades, las cuales han tomado diversidad de medidas para fortalecer la escritura académica en los diversos niveles educativos. Entre estas disposiciones, considero importante la asociación que tienen las distintas universidades brasileñas entre sí, así como sus alianzas con otras universidades, a nivel internacional, para la realización de actividades variadas en torno al tema de la escritura académica, tales como: trabajos de investigación colectiva, talleres, congresos, conferencias, encuentros, entre otros.

\section{ALGUNAS BASES TEÓRICAS}

Las ideas planteadas en esta investigación acerca de la escritura académica, como se mencionó anteriormente, se fundamentan en el enfoque pragmático textual, la teoría de la lingüística textual y el análisis del discurso, en el sentido de que la producción de un texto escrito retoma tanto elementos oracionales como aspectos transoracionales. Por tal razón, en este apartado se presentan algunos fundamentos que sustentan estas teorías, en general, así como aspectos relacionados con la escritura académica, en particular. 


\section{La escritura académica}

La escritura académica es una habilidad que se desprende de la expresión escrita, en general, puesto que, dentro de esta última existen otros tipos de escritura, tal como la escritura creativa. La expresión escrita, a la vez, es una de las habilidades que conforman la competencia comunicativa. Cada una de ellas requiere de ciertas exigencias, según la naturaleza del texto que se pretende producir. Al referirse a la escritura, en general, Sánchez Lobato (2007, p. 59) define el acto de escribir como "un proceso complejo que exige pensar, evaluar y modificar constantemente un escrito hasta lograr la forma definitiva. La escritura presupone el acto de reescribir. Solo de esta manera podemos lograr que un escrito sea adecuado, efectivo, coherente y correcto".

En tal sentido, para la producción de un texto académico el autor debe tener conocimiento sobre aspectos ineludibles para desarrollar la escritura académica. En primer lugar, debe conocer el tema por desarrollar, entre otros aspectos, tales como saber qué es un texto y qué lo define como tal. Al respecto, Calsamiglia y Tuson (1999, p. 119) consideran que "el texto es una unidad comunicativa de un orden distinto al oracional; una unidad semántico-pragmática de sentido, y no sólo de significado; una unidad intencional y de interacción, y no un objeto autónomo", pues del texto se valora lo dicho y lo no dicho, ya que tanto en su producción como en su comprensión, influyen los distintos tipos de contextos (lingüístico, cognitivo, situacional, social, cultural, entre otros).

Asimismo, es importante que los estudiantes universitarios, así como los alumnos de todos los niveles educativos, conozcan y comprendan cada una de las cualidades que debe cumplir un texto, entre ellas, la intencionalidad o propósito comunicativo, la coherencia, progresión temática, unidad temática, cohesión, adecuación, gramaticalidad, intertextualidad e informatividad. Además, debe tener conocimiento sobre las características particulares de cada tipo y modelo de texto, tanto estructurales como de contenido.

En vista de lo anterior, es fundamental conocer los modos de organización del discurso, los cuales responden y están, estrechamente, relacionados con las 
operaciones discursivas y las destrezas de narrar, describir, argumentar, explicar, entre otras. Así, dentro de los modos discursivos están: la narración, la descripción, la argumentación, la exposición, el diálogo; así como los modelos textuales que caben dentro de cada uno de ellos. En la escritura académica cobra particular importancia la argumentación, por lo tanto, un buen productor textual debe saber argumentar, con el fin de llamar la atención de su lector o interlocutor, tal como lo afirman Plantin y Muñoz (2011, p. 13) "argumentar consiste en dirigir a un interlocutor un argumento, es decir, una buena razón, para hacerle admitir una conclusión y, por supuesto, los comportamientos adecuados".

De igual manera, se debe tener conocimiento y dominio acerca de los diferentes procedimientos de cohesión textual, los cuales son mecanismos que sirven para establecer relaciones semánticas entre segmentos textuales, estos pueden ser procedimientos léxicos o gramaticales, entre ellos, se encuentran los siguientes:

\section{Procedimientos de cohesión textual}

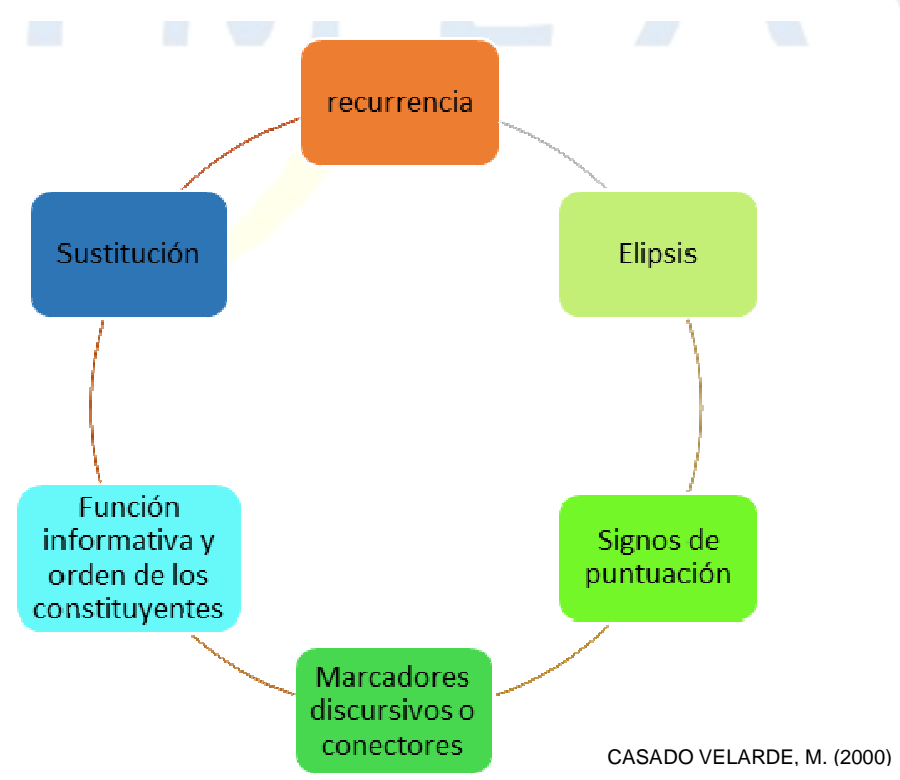

Además de los factores mencionados anteriormente, para la escritura académica es de suma importancia, el conocimiento y cumplimiento de las 
diversas fases para la producción de un texto escrito, es decir, la construcción del proyecto de escritura o planificación del escrito, la redacción, la revisión y la edición. También, es relevante reflexionar acerca de los elementos que comprende cada fase y considerarlos para tener éxito en la producción del texto escrito.

Competencias para una escritura académica adecuada

La competencia comunicativa se puede describir como una macrocompetencia, en el sentido de que su dominio implica el conocimiento de muchos aspectos que influyen, directamente, en nuestro desenvolvimiento en los distintos tópicos de nuestra vida diaria. Por lo tanto, es importante destacar que el fortalecimiento de la competencia comunicativa, desde los diversos ángulos y niveles educativos, a través de las competencias que la conforman, es decir, la competencia lingüística, la competencia discursivo-textual, la competencia ideológica, la competencia pragmática, la competencia cultural, entre otras, debe ser un proceso continuo y extensivo a las diferentes áreas del conocimiento, tanto en su tratamiento como competencia genérica, así como competencia específica, según sea el caso.

Para tal efecto, es necesario que se realice un trabajo colaborativo por parte de los distintos entes implicados en la tarea, es decir, estudiantes, docentes, expertos, así como las instituciones educativas mismas. Las competencias que integran la competencia comunicativa se complementan unas a otras, por lo tanto, funcionan de manera superpuesta, tal como se presentan en la figura. 


\section{Modelo de constitución de la competencia comunicativa}

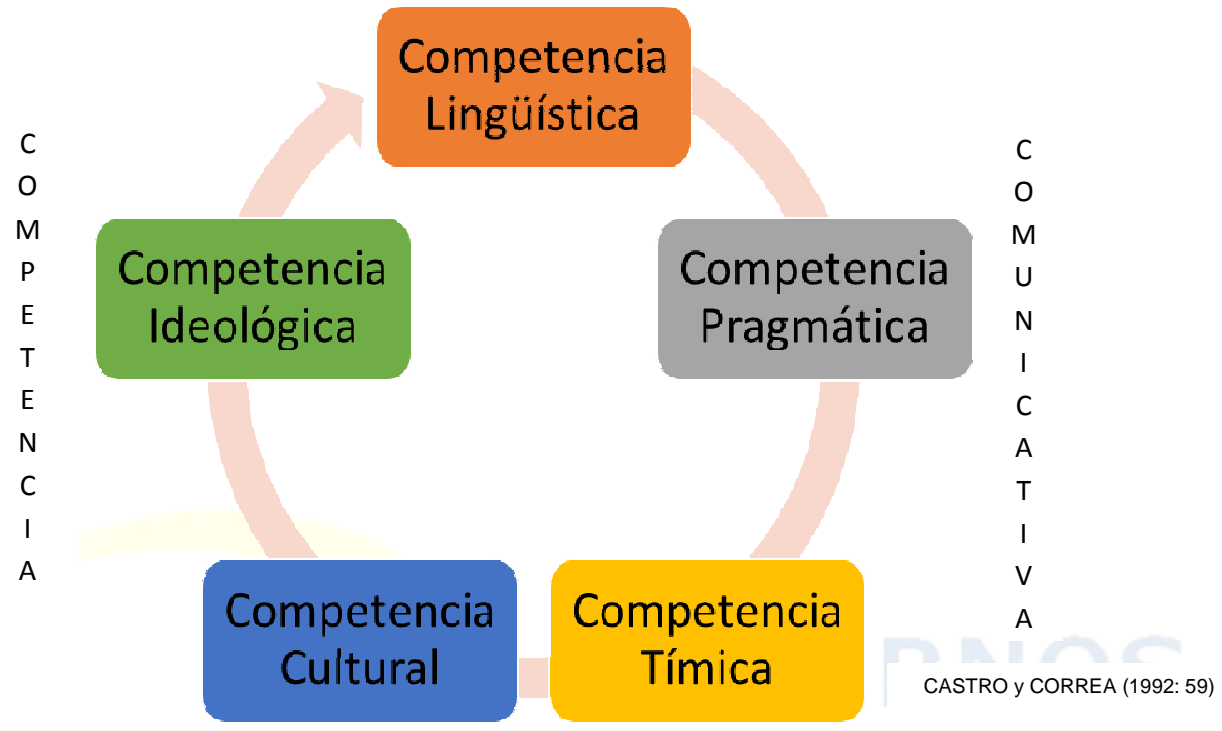

La escritura académica en la universidad

Es importante señalar, que la debilidad en cuanto a escritura académica es un aspecto bastante general en el ámbito universitario, tanto a nivel de pregrado como de los postgrados, no solamente en Honduras, sino en diversos países de Latinoamérica. En vista de que, a pesar de que la habilidad de escritura es considerada una competencia genérica, desde el punto de vista del enfoque por competencias, adoptado por la UPNFM, no todos los estudiantes de los diversos niveles y áreas del saber demuestran fortaleza en la misma. Por consiguiente, sus producciones escritas no siempre evidencian las cualidades o propiedades discursivo-textuales requeridas, así como las características pertinentes, según el tipo de texto y modelo textual que se produzca; para que este sea valorado como tal. En un estudio sobre escritura académica en el postgrado, realizado en la Universidad Pedagógica Nacional (UPN), de México, se refleja lo siguiente:

En el diagnóstico se detectaron dificultades temáticas, discursivas y lingüísticas. Dentro de las primeras, comentan su desconocimiento sobre el tema: "empíricamente lo he vivido" y la búsqueda de fuentes documentales: 


\begin{abstract}
"además, nadie me dice dónde y cómo buscar". Las dificultades discursivas: "no sé qué tipo de escrito es, ni cómo estructurarlo", "la forma de ir articulando párrafos en un escrito", "cuál es el propósito del escrito", "no sé cómo argumentar y qué tipo de argumentos usar", "cómo conectar las ideas en los párrafos". Como problemas lingüísticos, donde los estudiantes presentan dificultades gramaticales como la acentuación, ortografía y puntuación. Un alumno comentó que "escribir me provoca angustia". (CRUZ MARTÍNEZ, 2014-2015, p. 53).
\end{abstract}

En vista de lo anterior, se evidencia, a la vez, que tienen debilidad no solo en la expresión escrita, sino en la comprensión lectora y las distintas destrezas y microhabilidades que comprenden las mismas, pues ambos procesos están estrechamente relacionados, puesto que para ser un escritor capacitado, primero se debe ser un buen lector, tal como lo afirma Cassany, et al. (1997, p. 262) "los escritores competentes son buenos lectores o lo han sido en algún período importante de su vida. La lectura es el medio principal de adquisición del código escrito". En tal sentido, la lectura nos permite fortalecer diversos procesos, desde criterios mecánicos hasta aspectos cognitivos, imprescindibles para la producción del conocimiento a través de la escritura académica.

Por su parte, Flores, (2014, p.106), en la misma línea de pensamiento, afirma que "produzir um texto implica não somente escrevê-lo, sem dúvida, mas também ler algo que foi produzido anteriormente. Por isso, um texto é uma cena enunciativa duplamente conjugada...". Por lo tanto, es necesario fortalecer en los maestrantes y en los estudiantes del nivel superior, en general, las prácticas de lectura y escritura y, de esta manera, promover y mejorar la producción de conocimiento.

En vista de lo anterior, se debe tener en cuenta que todas las habilidades básicas que conforman la competencia comunicativa, es decir, la comprensión y la expresión oral, así como la comprensión lectora y la expresión escrita, (estas dos últimas son las que nos interesan en este trabajo, debido a su interdependencia), por un lado, son procesos cognitivos complejos y continuos, que deben fortalecerse, a lo largo de toda la formación académica y profesional del ser humano, por otro lado, requieren del dominio de los conocimientos técnicodisciplinares, entre otros, puesto que, no precisamente, se puede aprender de la 
misma manera en las diferentes áreas del conocimiento, debido al lenguaje científico y técnico de cada disciplina.

En tal sentido, se debe considerar que el discurso tanto oral como escrito no es un fenómeno puramente lingüístico, sino que además, se considera un fenómeno cognitivo, social, cultural, ideológico, que va mucho más allá del uso de estructuras lingüísticas, por lo cual, se deben tener en cuenta los diversos elementos contextuales en los que se produce el discurso. Al respecto, Foucault, citado por Fischer, afirma que:

..o discurso ultrapassa a simples referência a "coisas", existe para além da mera utilização de letras, palavras e frases, não pode ser entendido como um fenômeno de mera "expressão" de algo: apresenta regularidades intrínsecas a si mesmo, através das quais é possivel definir uma rede conceitual que lhe é propia. (FISCHER, 2012, p. 75).

En cuanto al fortalecimiento de la competencia comunicativa, en general, en el común de los casos, en los diversos niveles educativos las habilidades básicas de lectura y escritura no se enseñan como una asignatura o espacio formativo en especial, o como un tema, en particular, sino como un eje transversal, lo cual es válido; sin embargo, no está demás reforzar, además, algunos aspectos específicos referidos a estas habilidades, de manera más concreta; tal es el caso de las cualidades o propiedades que debe tener un discurso, sea este oral o escrito; los procedimientos de cohesión textual, cuáles son, en qué consisten y cómo utilizarlos; las características particulares que debe tener un texto, atendiendo a su intención comunicativa y, por consiguiente, el tipo y modelo de texto al que pertenece. En cuanto al tratamiento del texto académico y la producción de conocimiento en la universidad, Barzotto afirma que:

Para tratar o texto acadêmico como dado na formação universitária, defendemos que "a formação de professores contemple um pouco de epistemologia, no sentido mais básico, ou seja, de proporcionar sempre momentos de reflexão a respeito da relação entre sujeito e objeto, com vistas à produção de conhecimento" (BARZOTTO, 2018, p. 65). 
Con respecto a lo anterior, en el caso particular de la UPNFM, solamente en el programa de Maestría en Enseñanza de Lenguas con Orientación en Español se contempla un módulo sobre expresión escrita y otro sobre comprensión lectora, en los cuales se abordan, de manera general, algunos enfoques de enseñanzaaprendizaje de la lengua, así como algunas estrategias de enseñanza-aprendizaje, entre otros aspectos imprescindibles para fortalecer estas habilidades.

\section{MARCO METODOLÓGICO}

Esta investigación se enmarca en el enfoque cualitativo, es de tipo diagnóstica, exploratoria, descriptiva. Asimismo, se basa en el diseño emergente. La población participante está conformada por maestrantes de diversos programas de maestría de la FAHU/UPNFM. El corpus para este estudio fueron diez artículos científicos, escritos por los maestrantes, a partir de su tesis de grado, como requisito de culminación de su programa. En cuanto a la técnica de recolección de la información, los artículos fueron proporcionados a la investigadora por la Dirección de Postgrado de la UPNFM, después de haber solicitado una autorización, a través de la Rectoría, para poder usar los mismos. Para la revisión y análisis de los artículos, se utilizó una rúbrica de categorías y subcategorías referidas a diversos aspectos considerados para la producción y valoración de un texto escrito, la misma se construyó atendiendo a los objetivos de la investigación. Entre las categorías de análisis están: la intención comunicativa, adecuación del lenguaje al contexto de producción, coherencia textual, cohesión textual, intertextualidad, gramaticalidad, operaciones discursivas. La rúbrica se construyó con algunas ideas tomadas de Cáceres (2020, pp. 130-132).

\section{ANÁLISIS E INTERPRETACIÓN DE RESULTADOS}

En este apartado se dan a conocer, de forma resumida, los resultados del análisis e interpretación de los datos encontrados, a partir de diez artículos científicos. Es importante aclarar, que el análisis de los textos se fundamenta en el método de análisis textual, desde la perspectiva del análisis del discurso y del 
enfoque pragmático textual. El análisis textual se realizó de forma manual, el mismo consistió en una lectura detallada y exhaustiva, revisión, valoración y corrección de cada texto. Todo ello, con el fin de verificar el cumplimiento o no de las categorías y subcategorías de análisis contempladas en la rúbrica de evaluación, las cuales responden a diversos aspectos que contribuyen a la producción del discurso escrito.

Al respecto, es importante mencionar que, en algunos casos, los ejemplos de los hallazgos no fueron contextualizados con ejemplos de segmentos de textos analizados, debido a la restricción en la extensión del artículo. A continuación, se presenta un cuadro de análisis e interpretación de los resultados obtenidos:

\begin{tabular}{|c|c|c|}
\hline & 1. Intención comunicativa & Ejemplificación \\
\hline & $\begin{array}{l}\text { Con respecto a si los textos presentan un tema de } \\
\text { manera clara, se observa que varios de ellos tienen } \\
\text { claridad temática y desarrollan los elementos concernientes } \\
\text { al tema planteado en el título, en el caso del ejemplo 1, } \\
\text { considero que, a pesar de que tiene algunos elementos en } \\
\text { el título que no debería, a lo largo del artículo, desarrolla los } \\
\text { diversos aspectos referentes al tema del potencial turístico, } \\
\text { así que en este caso el autor tiene claro el tema por } \\
\text { desarrollar. } \\
\text { Sin embargo, en el ejemplo } 2 \text {, el tema planteado a través } \\
\text { de todos los elementos comprendidos en el título, es } \\
\text { demasiado vasto, pues habla de muchos aspectos que } \\
\text { podrían dar lugar a varios trabajos de investigación. Al } \\
\text { hablar de "competencias comunicativas", (por cierto, lo } \\
\text { adecuado es "competencia comunicativa"), esta integra } \\
\text { diversas habilidades y destrezas, es decir, la comprensión y } \\
\text { expresión oral, así como la comprensión lectora y la } \\
\text { expresión escrita. Además de estos, se mencionan otros } \\
\text { elementos en el título. Por lo tanto, todos ellos deberían de } \\
\text { estudiarse en la investigación y estar desarrollados, de } \\
\text { manera coherente, en los diversos apartados que } \\
\text { comprende el proceso de investigación y, por consiguiente, } \\
\text { del artículo, el cual fue producido a partir de su tesis de } \\
\text { grado. No obstante, en este, el autor solamente menciona } \\
\text { la escritura creativa y la comprensión lectora, de forma muy } \\
\text { general. En consecuencia, este es uno de los riesgos que } \\
\text { se corre al tener un título que no esté bien delimitado, que } \\
\text { no tenga claridad y en el cual no se expresen, de manera } \\
\text { puntual, los elementos que se desea investigar. Por } \\
\text { consiguiente, el resultado es una investigación muy }\end{array}$ & $\begin{array}{l}\text { Ejemplo } 1 \\
\text { "Potencial turístico } \\
\text { en el desarrollo } \\
\text { local de la aldea } \\
\text { de Cedeño, } \\
\text { municipio } \\
\text { Marcovia en el } \\
\text { periodo 2014-2018" } \\
\text { Ejemplo } 2 \\
\text { Desarrollo de las } \\
\text { competencias } \\
\text { comunicativas en } \\
\text { los bachilleratos } \\
\text { humanístico } \\
\text { técnico y } \\
\text { profesional en } \\
\text { informática desde } \\
\text { la perspectiva del } \\
\text { trinomio estudiante- } \\
\text { docente-currículo }\end{array}$ \\
\hline
\end{tabular}




\section{Intención comunicativa}

superficial y vaga.

1.2. En cuanto a si el texto tiene un propósito comunicativo bien definido, este es un aspecto que va de la mano con el título y, por consiguiente, con el tema, en general. En tal sentido, en aquellos textos en los cuales el título y el tema están bien definidos, se percibe que el propósito comunicativo también los está, por el contrario, aquellos que presentan títulos o temas mal planteados divagan en su propósito comunicativo.

\begin{tabular}{|l|l|}
\hline 2. Adecuación del lenguaje al contexto de producción & Ejemplificación \\
\hline 2.1. $\begin{array}{l}\text { En lo que se refiere a si el texto presenta un lenguaje } \\
\text { adecuado a su lector y al contexto de producción, en }\end{array}$ & \\
general, los escritos presentan un lenguaje adecuado, \\
sobre todo, adecuado al área de disciplinar, pues se usan \\
tecnicismos propios de cada especialidad de las maestrías, \\
sin embargo, en un caso particular, hay confusión en el uso \\
de algunos términos.
\end{tabular}

\section{Coherencia textual}

3.1. En lo referente a si el texto muestra un título bien delineado y adecuado al tema, de los diez textos solamente uno de los artículos tiene un título bien definido, que cumple con los requerimientos necesarios y que deja bien claro lo que se quiere investigar, es el caso del primer ejemplo. El resto de los artículos presentan títulos demasiado extensos, puesto que en ocho de ellos, se incluye en el título aspectos concernientes a la delimitación de la investigación, especialmente, en lo referente a la delimitación espacial y temporal.

Lo anterior, por un lado, limita la dimensión de la investigación, de alguna manera, ya que si por ejemplo se investiga sobre producción escrita, aunque la investigación se realice en cierto espacio o institución, el impacto (no los resultados) de la misma bien puede ser válido para muchas otras instituciones educativas, como es el caso del segundo ejemplo.

Por otro lado, en algunos casos el título no está bien definido y es muy extenso, no en cuanto a su estructura, sino a la dimensión de su alcance; lo que puede conducir a dos escenarios: uno, la investigación puede hacerse de forma muy superficial, dos, la investigación puede requerir demasiado tiempo para cumplir con todo lo dicho en el título. En el caso del tercer ejemplo, existen muchas estrategias de evaluación, según
Ejemplificación

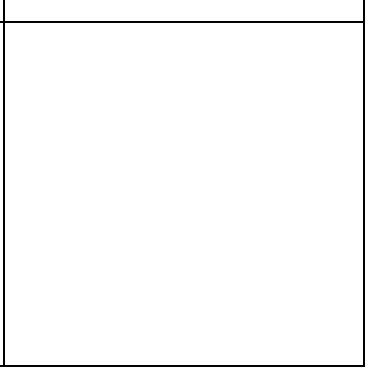

Ejemplificación

Ejemplo 1

La Letra de Canciones como Recurso

Didáctico para

Potenciar la

Comprensión Lectora y la Expresión Escrita

\section{Ejemplo 2}

El Cuento como Estrategia Didáctica para Mejorar la Producción Escrita (en el Instituto Ángel G. Hernández)

\section{Ejemplo 3}

Prácticas Pedagógicas y Estrategias de Evaluación, (en el Centro Universitario de Educación a Distancia, de la Universidad

Pedagógica Nacional Francisco Morazán). 


\section{Coherencia textual}

diversos tipos y enfoques, por lo tanto, se debe precisar sobre cuál se quiere investigar. Además, la UPNFM tiene siete Centros Universitarios de Educación a Distancia (CUED), si se plantea de manera tan general, significa que se deben estudiar los siete centros. Por tal razón, es importante que este dato no vaya en el título, sino en la delimitación espacial de la investigación, en donde se especifique en cuál CUED se hará el estudio. En el otro artículo se habla de "competencias comunicativas", es decir, en plural, sin embargo, tenemos una competencia comunicativa, que integra diversas habilidades y destrezas. No es adecuado referirse en plural.

Además de los aspectos mencionados anteriormente, algunos títulos de los artículos también incluyen, en su estructura, la población participante de la investigación, como en el tercer ejemplo.

3.2. Con relación a si el texto presenta introducción clara del tema, todos los artículos tienen una introducción, puesto que obedecen a una estructura dada por la Dirección de Postgrado para la escritura de los mismos. No obstante, algunos de los textos analizados presentan problemas de redacción, y, en ciertos casos, la introducción no cuenta con los elementos necesarios que esta debe cumplir, tales como el tema y el propósito u objetivos del estudio.

Ejemplificación

Ejemplo 3

La Resolución de

Conflictos como

Estrategia de Mejora

de la Convivencia

Escolar

Estudiantes

en

Docentes (del Noveno

Grado Sección "A" del

Centro Educativo

Santa María Goretti).
3.3. En lo que se refiere a si el texto evidencia desenvolvimiento o progresión temática, esta es una condición muy relacionada con la unidad temática, en tal sentido, los resultados frente a estos aspectos son similares, en general, en atención a la estructura dada por la Dirección de Postgrado para la construcción de los artículos, estos muestran una secuencia y avance en la exposición de las ideas, para lo cual se auxilian de algunos tipos de marcadores discursivos como los tematizadores que indican el avance según el tópico desarrollado en cada apartado o párrafo, como en el ejemplo 1. Sin embargo, en algunos casos se pierde la progresión, puesto que las ideas están expuestas de manera inconclusa y hay una ruptura abrupta, hay párrafos desconectados, como es el caso del texto que en su título expone algo distinto a lo que plantea en el desarrollo del texto, como sucede en el ejemplo 2 , entre otros.

Ejemplo 1

La Letra de Canciones como

Didáctico

Potenciar

Recurso

para

Comprensión Lectora y la Expresión Escrita

\section{Ejemplo 2}

La Comprensión

Lectora en el Primer

Curso de Ciclo Común

(del Instituto Manuel

Bonilla del Municipio

de Apacilagua, Choluteca).

\section{Ejemplo 1} atención a la estructura dada por la Dirección de La Comprensión
Postgrado para la construcción de los artículos, en Lectora en el Primer general, la mayoría de estos evidencian un hilo Curso de Ciclo Común 


\section{Coherencia textual}

conductor entre los diversos apartados que comprenden. Sin embargo, algunos presentan ideas inconclusas que hacen que se pierda el sentido del texto. Asimismo, uno de los artículos presenta un título (el que aparece en el ejemplo1), que, además de que está mal planteado; al desarrollar el artículo, lo describe como algo diferente, da la impresión de que está presentando otro trabajo de investigación, pues, a lo largo del texto, se refiere a: “El Taller Educativo como estrategia didáctica para mejorar la comprensión lectora en el primer curso de ciclo común del Instituto Manuel Bonilla del municipio de Apacilagua, Choluteca".

Asimismo, algunos de los artículos presentan ideas inconclusas o confusas. En el ejemplo 2 hay confusión en el uso de los términos "recursos y estrategias". Además, presenta errores de ortografía y de concordancia, como en los marcados.

3.5. En lo referente a si el texto presenta conclusión adecuada, en todos los artículos se presentan conclusiones, dado que responden a la estructura estipulada por la Dirección de Postgrado para la presentación de los mismos. La mayoría de los artículos, a pesar de que algunos tienen elementos de más en el título, presentan conclusiones adecuadas al tema desarrollado, no obstante, aquellos que tienen un título no bien definido y, por lo tanto, el tema no es claro, presentan conclusiones inadecuadas o pobres.

\section{Ejemplificación}

(del Instituto Manuel Bonilla del Municipio de Apacilagua, Choluteca).

\section{Ejemplo 2}

"no obstante, aún prevalece la estrategia tradicional de pizarra y marcador, ello supone aun la presencia de estrategia tradicional para la enseñanza, en cuanto a la ayudas y recursos didácticos se refiere".

\section{Cohesión textual}

\subsection{Con respecto a si el texto evidencia un uso Ejemplo}

adecuado de los procedimientos de cohesión Entre los marcadores textual, se observa que los más utilizados son los discursivos y conectores marcadores discursivos y conectores y los signos de puntuación. Ambos procedimientos, en su mayoría, fueron bien utilizados.

Con relación a los marcadores discursivos y conectores, en general, estos fueron utilizados, según su clasificación y, de acuerdo con sus distintas funciones y gamas, como se observa en el ejemplo. A pesar de ello, en ciertos casos, estos fueron utilizados de manera errónea, en el sentido de alterar su forma, por ejemplo se utiliza "a su vez" (no debe usarse el adjetivo posesivo "su") en lugar de su forma adecuada "a la vez", pues este cumple la función de marcador discursivo temporalizador expresivo de simultaneidad $y$, en ningún momento, indica posesión. Asimismo, en el uso de los distribuidores, utilizados, sobresalen los siguientes, se enumeran solamente algunos, a manera de ejemplo:

a. Aditivos (y, también, además, asimismo).

b. Continuativos (pues, por tal razón, por las razones antes expuestas, por 10 cual, en ese sentido).

c. Causales (ya que, porque, puesto que, debido a que, dado que, en vista de que). 


\section{Cohesión textual}

el error más común es que solamente utilizan uno de ellos, y, al ser distribuidores de la información, el uso de uno, presupone el uso del otro, funcionan en par, ejemplo: por un lado...por otro lado. Otro factor evidente es el uso reiterado de algunos conectores y marcadores discursivos, lo que demuestra el desconocimiento, por parte de los maestrantes, de que existen gamas de ellos que son intercambiables, puesto que cumplen la misma función. Especialmente, repiten en exceso los aditivos ( $y$, además, también), el conector causal (ya que), el tematizador (en cuanto a), lo que causa cacofonía o monotonía en el texto.

Con respecto al uso de los signos de puntuación, en general, fueron bien utilizados, sin embargo, uno de los signos en los que presentan mayor debilidad, en cuanto a su uso, es la coma, ya que, en ciertos casos, la usan donde no se debe o está ausente donde debe usarse. Por ejemplo:

a. Separan el sintagma nominal del sintagma verbal por una coma, lo que es inadecuado.

b. Algunos conectores, como los explicativos o reformuladores, deben ir entre comas; lo mismo ocurre después de los marcadores discursivos, siempre debe colocarse una coma, pero no la usan.

c. Las frases explicativas, así como los modificadores oracionales deben ir entre comas, aunque en muchos casos no las colocan.

d. Otro error común que encontré fue el uso de inicial mayúscula después de usar el punto y coma, sin haber una palabra que lo amerite, según las reglas. En resumen, en todos los casos de uso inadecuado de cualquier signo de puntuación, se corre el riesgo de que se altere el sentido del texto, pues las ideas lucen incompletas, son entrecortadas o confusas.

\section{Ejemplificación}

d. De consecuencia (por consiguiente, por lo tanto, de manera que).

e. Topicalizadores 0 tematizadores (en lo referente $a$, con relación a, en cuanto a, con respecto a).

f. Expresivos de punto de vista (según..., por su parte, de acuerdo con, desde diversos puntos de vista).

g. Explicativos reformuladores (es decir, o sea).

h. Contrastivos contraargumentativ os (sin embargo, sino, mientras que, pero, no obstante, aunque).

i. De finalidad (a fin de que, con el fin de que).

j. Temporalizadores (a la vez, al mismo tiempo).

k. De certeza (con propiedad, definitiva, sin duda).

. Distribuidores (por un lado/por otro lado).

\section{Intertextualidad}

5.1. Con relación a si el texto presenta citas o referencias directas de otros escritos, todos los artículos evidencian el uso de citas directas, en la mayoría de los casos están bien utilizadas, en lo referente a su conexión con el contenido del texto, a su extensión, y a los datos de referencia, asimismo, en cuanto al uso de marcadores discursivos expresivos de opinión o punto de vista. A pesar de ello, en ciertos casos no se marcan, de manera adecuada, los signos gráficos que las delimitan, es decir, se sabe dónde inician, pero no dónde

\section{Ejemplificación}

\section{Ejemplo}

Asimismo, Ochoa y Achugar (2010, p. 49), Sostienen que la coherencia: "Se refiere al significado global del texto y a su efecto como unidad como resultado de la correlación entre las 


\begin{tabular}{|c|c|c|}
\hline & $\begin{array}{l}\text { terminan porque no cierran las comillas. En otros casos, } \\
\text { es evidente que son citas, pero no las marcan entre } \\
\text { comillas. Otro aspecto que preocupa es que hay textos } \\
\text { que tienen demasiadas citas, hay párrafos completos } \\
\text { que son una cita y no tienen ningún aporte de } \\
\text { producción de conocimiento, por parte del autor, como } \\
\text { en el ejemplo, este es un párrafo completo. }\end{array}$ & $\begin{array}{l}\text { características } \\
\text { internas y externas del } \\
\text { texto." }\end{array}$ \\
\hline 5.2. & $\begin{array}{l}\text { En cuanto a las citas o referencias indirectas de otros } \\
\text { textos, al igual que el caso de las citas directas, se } \\
\text { utilizan en todos los artículos. También, en muchos } \\
\text { casos se evidencia el mismo problema, citas demasiado } \\
\text { extensas y muy poca producción por parte del autor, } \\
\text { como en el ejemplo, este se presenta como un párrafo } \\
\text { completo, pero no hay desarrollo de las ideas. }\end{array}$ & $\begin{array}{l}\text { Ejemplo } \\
\text { Según Aristóteles } \\
\text { cuando tenemos un } \\
\text { texto se comprende lo } \\
\text { conocido, lo } \\
\text { contrastante } \quad \text { o } \\
\text { diferente y lo contiguo. }\end{array}$ \\
\hline
\end{tabular}

\begin{tabular}{|c|c|c|}
\hline & 6. Gramaticalidad & Ejemplificación \\
\hline 6.1 . & $\begin{array}{l}\text { En lo que se refiere a si el texto evidencia un uso } \\
\text { adecuado de los criterios de gramaticalidad, se } \\
\text { valoró el uso de aspectos fonológicos, } \\
\text { morfológicos, sintácticos y textuales, tales como la } \\
\text { concordancia de género, número y persona, entre } \\
\text { otros. Al respecto, en general, están bien } \\
\text { trabajados estos criterios, pues varios de los } \\
\text { artículos analizados fueron escritos por estudiantes } \\
\text { de la Maestría en Enseñanza de Lenguas. Sin } \\
\text { embargo, algunos de los artículos, sobre todo, } \\
\text { aquellos que fueron escritos por estudiantes de } \\
\text { otras maestrías, presentan problemas de diversa } \\
\text { índole referidos al cumplimiento de la } \\
\text { gramaticalidad, entre ellos: en el nivel fonológico, } \\
\text { se observan problemas de acentuación, } \\
\text { especialmente, cuando se trata de la acentuación } \\
\text { diacrítica ejemplo } 1 . \text { Asimismo, uso inadecuando } \\
\text { de las pausas, al no usar, apropiadamente, los } \\
\text { signos de puntuación, lo cual altera la curva de } \\
\text { entonación, y, por lo tanto, el sentido del texto, al } \\
\text { realizar la lectura del mismo. } \\
\text { En el nivel morfológico, se evidencian algunas } \\
\text { dificultades tales como: uso inoportuno del } \\
\text { gerundio, lo usan para iniciar un párrafo (ejemplo } 2 \text {, } \\
\text { este se presenta como un párrafo completo, sin } \\
\text { sentido y desconectado del anterior), también, usan } \\
\text { el gerundio como conector, lo cual es inadecuado. } \\
\text { Otro aspecto es la confusión en cuanto al uso de } \\
\text { algunas preposiciones, de acuerdo con su función, } \\
\text { pues confunden los contextos en donde debe ir una } \\
\text { u otra. (a/con), (de/por), (para/por), ejemplo } 3 \text {. }\end{array}$ & 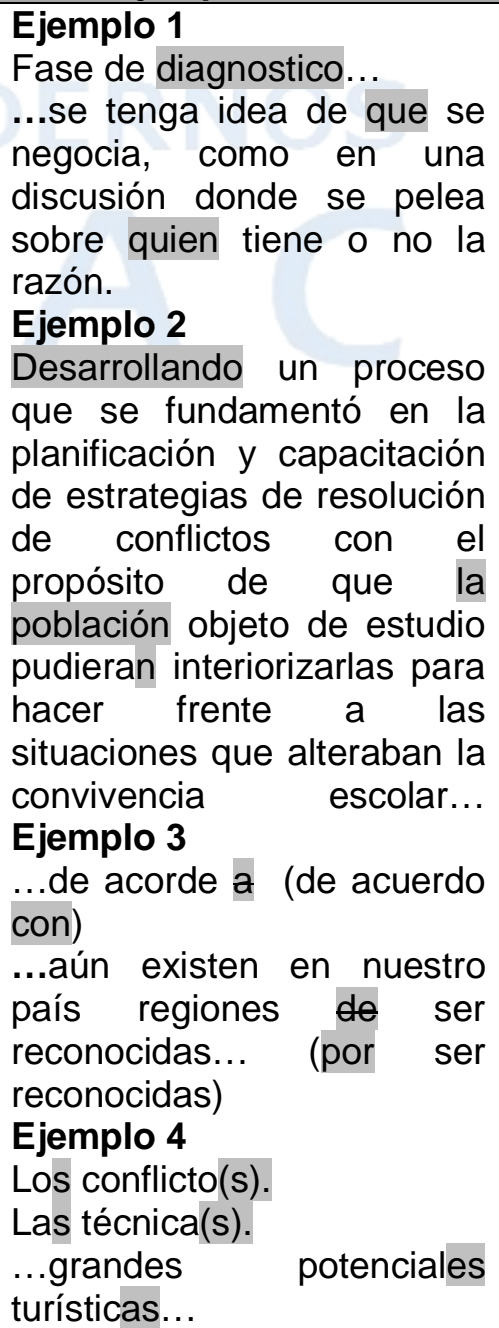 \\
\hline
\end{tabular}




\section{Gramaticalidad}

En el plano sintáctico, también, se evidenciaron algunas debilidades acerca de la concordancia de número y de género entre los elementos, ejemplo 4. Además, de alteraciones en el orden sintáctico, así como oraciones con un sentido incompleto.

Además de los aspectos mencionados anteriormente, se encuentran otros problemas que afectan la escritura, como son: el uso de siglas sin precisar su significado, lo cual es fundamental para que cualquier lector comprenda su significado. Asimismo, algunas siglas las usan en plural, lo cual no es aceptable, desde el punto de vista de la Real Academia Española de la Lengua (RAE, 2011, p. 583), ejemplo 6.

\section{Ejemplificación}

\section{Ejemplo 5}

De acuerdo con Zabalza (2002: p.97-103), la evaluación cuantitativa basada en la observación, medición, cuantificación y control.

\section{Ejemplo 6}

ONGS

\section{Operaciones discursivas Ejemplificación}

7.1. En lo concerniente a si el Ejemplo 1

texto presenta diversos modos de organización del discurso escrito, son diversas las operaciones discursivas representan estos modos en los artículos analizados. Al tratarse de artículos científicos, especialmente, Una de las actividades académicas en las que mayor provecho puede generar el empleo de canciones como recurso didáctico es la redacción de ensayos, pues al utilizar el argumento de una canción como idea temática, se promueven procesos cognitivos que se traducen en la redacción de textos inéditos de niveles satisfactorios.

\section{Ejemplo 2}

Potencial turístico: es el aprovechamiento de los encuentra las siguientes:

a. La argumentación, ejemplo 1.

recursos y potencialidades que tienen los habitantes de un espacio geográfico, con el propósito de utilizarlos de manera...

b. La definición, ejemplo 2.

Ejemplo 3

c. $L a$ ejemplo 3.

d. La comparación, ejemplo 4.

Por su (la) ubicación geográfica que posee la aldea de Cedeño limita con fronteras marítimas y terrestres. La carretera principal que conduce de Choluteca a la aldea de Cedeño esta (está) pavimentada...

\section{Ejemplo 4.}

e. La explicación, ejemplo 5.

f. La clasificación, ejemplo 6.

Además de estas, se encuentran otras, como la explicación, ejemplificación, etc. En las diversas operaciones utilizan, en gran medida, los marcadores utilizarse como cualquier otro texto ora para desarrollar la comprensión auditiva y como cualquier texto escrito para fomentar la comprensión lectora...

Ejemplo 5

En otras palabras, los estudiantes deben crear autonomía y desarrollar el hábito de la lectura y escritura...

\section{Ejemplo 6}

- Material construido por el alumnado y/o el profesorado... 


\section{Operaciones discursivas}

discursivos o conectores expresivos de cada una de ellas.

\section{Ejemplificación}

- Material tomado de la vida cotidiana...

- Material de desecho...

\section{CONCLUSIONES}

1. Al analizar y valorar si las producciones escritas por los maestrantes de la UPNFM cumplen con las diversas propiedades o cualidades discursivotextuales como son: la intención comunicativa, la adecuación, la coherencia (unidad temática y progresión temática), la cohesión, la intertextualidad y la gramaticalidad; se observa que las mismas no son reflejadas plenamente, pues, aunque, algunos textos evidencian que sus autores conocen acerca de estas propiedades, la mayoría de los escritos adolecen de ellas, en distintos sentidos. Esto es muy preocupante, puesto que no solo se trata de evaluar a los maestrantes, autores de estos textos, en vista de que los mismos fueron presentados ante la Dirección de Postgrado, como requisito de graduación de su programa de maestría, lo que significa que fueron revisados y aprobados por sus revisores. En tal caso, es importante también, la asignación de revisores competentes y la evaluación de su desempeño en esta tarea tan importante, pues además de revisar, se debe corregir y orientar al estudiante, ofrecerle estrategias de solución cuando este presenta debilidades.

2. En cuanto al tipo de relación que los maestrantes establecen respecto a la citación de otros autores, en sus producciones escritas; al ser la escritura un proceso complejo, este requiere del conocimiento del tema acerca del cual se escribe, pues no se puede argumentar sobre algo desconocido. En tal sentido, un buen productor de textos, primero debe ser un buen lector, pues esto acrecienta su conocimiento y le permite establecer relaciones entre lo que produce y lo que ha leído, previamente, sobre el tema, de modo que, lectura y escritura van de la mano. Por lo tanto, la citación es un procedimiento obligado. Los textos analizados evidencian el uso de citas tanto directas como indirectas, en ciertos casos, el uso de las mismas es adecuado, sin embargo, 
en otros, las citas son demasiado extensas y hay muy poca producción por parte del autor. asimismo, es evidente que algunos segmentos de texto son citas, pero no las marcan entre comillas, quizá de manera consciente o inconsciente, lo cual, de alguna forma, conduce al plagio. Otro factor preocupante es que hay textos que tienen demasiadas citas (párrafos completos), y muy poco o ningún aporte de producción de conocimiento, por parte del autor. En consecuencia, es fundamental fortalecer este aspecto para optimizar la escritura académica en el postgrado.

3. Con respecto a las diversas operaciones discursivas necesarias para la producción de un texto escrito, los maestrantes utilizan diferentes modos para organizar el texto, entre ellos: la argumentación, la definición, la descripción, la comparación, la explicación, la clasificación, la explicación, la ejemplificación, entre otros. Es importante mencionar, que para la construcción de las diversas operaciones discursivas, ellos utilizan los distintos marcadores discursivos y conectores; en tal sentido, evidencian tener conocimiento al respecto, a pesar de que algunas veces, tanto los modos discursivos como los marcadores discursivos y conectores que los identifican no se utilicen de manera adecuada, en su totalidad.

4. Los artículos escritos por los maestrantes evidencian tanto fortalezas como debilidades en cuanto a la capacidad para la escritura académica, y, por consiguiente, para la producción de conocimiento. Las debilidades son muchas, y se enmarcan en los diversos aspectos que intervienen en la expresión escrita, pues como bien lo expresa Sánchez Lobato, escribir es un proceso complejo, por lo que requiere de un vasto conocimiento desde factores mecánicos hasta cognitivos.

En general, con respecto a lo anterior, todos los factores se resumen en el dominio de las diversas competencias que conforman la competencia comunicativa, o sea, si retomamos lo planteado en la figura sobre la constitución de esta competencia, la misma está integrada por: la competencia lingüística, es decir, que para saber escribir se requiere el dominio profundo de la lengua en la 
cual se escribe, en todos sus niveles de análisis. Asimismo, la competencia pragmática, esto es, el cómo usar la lengua de acuerdo con la situacionalidad de enunciación, es decir, saber adaptar el discurso escrito a los distintos tipos de contextos que lo condicionan. También, la competencia ideológica, puesto que para producir un texto se debe adoptar posturas mentales acerca de teorías, enfoques, autores. Además se menciona la competencia cultural, pues la lengua en sí misma es un elemento de cultura y la tímica que influye en la interacción comunicativa entre el productor y el interlocutor, todas ellas conducen a la competencia discursivo-textual. En tal sentido, es una necesidad fortalecer la escritura académica en la universidad, en los estudiantes de todos los niveles. Por lo tanto, las autoridades universitarias deben tomar serias decisiones, al respecto.

\section{RECOMENDACIONES}

Considerando los hallazgos de esta investigación, así como algunas vivencias tanto desde del ámbito docente como del estudiante es importante reflexionar acerca de la adopción de ciertas medidas que contribuyan al fortalecimiento de la escritura académica en el nivel de postgrado y, por consiguiente, en la producción de los trabajos de tesis que cada maestrante debe presentar como requisito de graduación en la mayoría de universidades y, de esta manera, lograr un mejor nivel de eficiencia terminal, puesto que, en aquellos casos en los cuales los maestrantes rezagan su trabajo de tesis, por diversas razones, entre ellas, la dificultad en la expresión escrita, la eficiencia terminal es uno de los aspecto que se ve más afectado, quizá de manera indirecta. Por tal razón, se presentan las siguientes recomendaciones, válidas para diversos programas de maestría, en general:

Toda institución universitaria debe brindar acompañamiento, de manera permanente, a cada maestrante, desde el inicio hasta el final de su programa. En tal sentido, se debe considerar la posibilidad de asignar orientadores especialistas, en las temáticas seleccionadas para sus trabajos de tesis. 
Las universidades deben establecer alianzas estratégicas con universidades amigas tanto a nivel nacional como internacional, con el fin de conformar equipos de asesores especialistas en las diversas áreas disciplinares, así como asesores metodológicos y de redacción técnico-académica, de acuerdo con cada programa de maestría. Asimismo, para poder contar con equipos de ternas evaluadoras (igualmente, con docentes especialistas en el tema de tesis) para el momento de la defensa.

Al reflexionar acerca de que una de las principales causas de rezago en los programas de maestría es el hecho de que los maestrantes no logran escribir su trabajo de tesis, es importante reglamentar, de manera rigurosa, el proceso de eficiencia terminal, con el fin de establecer plazos específicos para el cumplimiento de cada etapa, y que estos plazos sean funcionales, tal como sucede en universidades de otros países. Como lo comenta Barzotto, respecto al caso de las universidades de Brasil, la eficiencia terminal está vinculada a:

- Existencia de una estructura para los posgrados, con secretaria, ambientes con computadoras, etc.

- Obligatoriedad de cumplimento de plazos. Si el alumno no termina su tesis, pierde el derecho de inscripción. Como todo el curso de posgrado es gratis, no hay porque mantener el estudiante inscrito si no está haciendo lo que debe hacer.

- La existencia de becas. No hay para todos, pero, cuando el estudiante tiene una beca debe culminar en el plazo establecido. Si no culmina, pierde el derecho a su inscripción y, en este caso, debe devolver el monto de la beca.

- La evaluación constante del estudiante, del director de tesis y del programa de posgrado. Todos pierden si el estudiante no termina.

- Hay muchos otros elementos que obligan a que se termine a tiempo. Por eso la gente busca escribir y superar las dificultades. 
Las universidades deben contar con un centro de escritura académica, o en su defecto, brindar talleres de escritura académica tanto para estudiantes de pregrado como de postgrado, en los distintos programas de maestría, con el propósito de brindar asesoramiento a quien así lo requiera.

\section{REFERENCIAS}

BARZOTTO, Valdir Heitor. Enseñanza de lectura y escritura: construyendo relaciones vitales entre el sujeto, su idioma y su cultural. Revista-Boletín Redipe, V-7, N 9, p. 65. Septiembre, 2018.

BLANCAFORT, Helena Calsamiglia y VALLS, Amparo. Las cosas del decir. Barcelona: Ariel, S.A.,1999.

CASSANY, Daniel; LUNA, Marta; SANZ, Gloria. Enseñar Lengua. Barcelona, España: Editorial GRAÓ, 1997.

DIRECCIÓN DE POSTGRADO, UPNFM. Informe de Investigación sobre Sistematización de la Experiencia del Proceso de Eficiencia Terminal en los Programas de Postgrado UPNFM, 2020.

ESTRADA, Sandra Cáceres. El discurso argumentativo escrito: su construcción desde los marcadores discursivos y conectores. Tegucigalpa, Honduras. Sistema Editorial Universitario (SEU/UPNFM), 2020.

FISCHER, Rosa María Bueno. Trabalhar com Foucault: arqueología de uma paixão. Belo Horizonte: Autêntica Editora, 2012.

FLORES, Valdir do Nacismento. Esnino, escrita, enunciação: uma questão de leitura. In FABIANO, Sulemi y BARZOTTO, Valdir, Heitor (Org.). Ensino da leitura e da escrita. Natal: EDUFRN, 2014.

LOBATO, Jesús Sánchez. Saber escribir. Madrid: Aguilar, 2007.

MARTÍNEZ, Ana Cruz. La atención de la escritura académica en el posgrado: una experiencia en la UPN. Revista de Investigación Educativa de la REDIECH, México, v. 9, 2015.

MEDINA, José Correa, RODRÍGUEZ, Cecilia y ÁLVAREZ, Nancy MARTíNEZ. Saber y saberlo DEMOSTRAR. Bogotá: Universidad Externado de Colombia, 1999. 
PLANTIN, Christian; MUÑOZ, Nora. El hacer argumentativo. Buenos Aires: Biblos, 2011.

REAL ACADEMIA ESPAÑOLA DE LA LENGUA (RAE). Ortografía de la lengua española. México: ESPASA, 2011.

SECRETARÍA UPNFM. Cuadros de registro de maestrantes de la Maestría en Enseñanza de Lenguas, 2021. 